\title{
New asset management structures for small- to medium-sized funds
}

Received: 12th June, 2000

\section{Debbie Harrison}

is an award-winning financial author and journalist. She is a regular contributor to the Financial Times, 'Bloomberg Money' and 'Investment Week'. Debbie specialises in UK and European pension fund issues and is the author of six Financial Times management reports on this subject. Her consumer titles include 'The Money Zone', 'Pension Power', 'First Time Investor' and 'Personal Financial Planner', all published by the Financial Times Prentice Hall.

\begin{abstract}
A recent Financial Times Management Report suggested that traditional asset management models may be failing to meet the needs of many trustees of small- to medium-sized schemes, which historically have not had the scope for sophisticated multi-manager, multi-style structures. The report argues that the UK market is now ready for providers who can offer these structures on a pooled basis at a competitive price. Furthermore, it suggests that some funds would benefit from a 'manager of managers' (MoM) service, where trustees outsource to a professional the decisions to hire, monitor and fire the managers.

However, it is still early days for the 'manager of managers' market. Before following this route, trustees must be satisfied that MoM is genuinely effective and not, as its critics suggest, just another over-priced, over-engineered American gimmick.

The acceptance of MoM for the mass market in the UK is conditional on five vital steps:
\end{abstract}

1. Providers should avoid the flawed assumption that the techniques used to monitor the style-specific managers in the US can be applied directly to UK managers.

2. The UK regulators should consider introducing a framework that allows trustees formally to outsource the job of appointing the asset managers to a professional.

3. Trustees who have limited time for asset management decisions should consider outsourcing to professionals the implementation of the asset management strategy and transition management to avoid unnecessary losses.

4. The pensions industry should relax its traditional view that asset management and investment consultancy are separate disciplines. MoM combines both and those who try to pigeonhole it into just one category are missing the point entirely.

5. Investment consultants should consider how MoM could help them to achieve the successful implementation of their recommended investment strategy. MoM can be the consultants' best friend and not, as some view it, a direct competitor.

Debbie Harrison (6 Palmers Hill, Epping Essex CM16 6SG Tel: $+44(0) 1992$ 577420)
Keywords: trustee dissatisfaction; manager of managers; outsourcing asset management; implementation; transition management; liability for performance 


\section{Introduction}

An asset management model, which relies on certain managers, sectors and styles being in favour at any particular time, is fundamentally flawed. Even with the most advanced risk modelling programmes, the providers are looking to the past to predict the future. Inevitably this can prove inefficient and misleading.

Given these uncertainties, trustees need to consider very carefully which type of management structure, manager review process and implementation procedures best demonstrate good investment governance under the Pensions Act 1995.

The FT Management Report 'Competitive multi-manager structures' argues that the small but growing number of providers who offer multimanager funds on a pooled basis are well placed to enter the market for group personal pensions and low-cost stakeholder schemes.

However, the report demonstrates that some consultants are not giving trustees of smaller funds sufficient information to enable them to make an informed choice between traditional and more modern asset management structures. In particular, trustee awareness of multimanager, multi-style and manager of managers is very low (see Appendix).

\section{Survey of trustee attitudes to investment management}

The report includes the results of a unique survey conducted by FT Management Reports and the Westminster Business School. The Pensions Management Institute (PMI) Trustee Group was used as a special control group within the survey.

Looking at the overall response, almost half of trustees (47 per cent) said they were not happy with the performance of their pension fund. Of this group, there is a greater proportion of dissatisfaction among trustees of smaller schemes

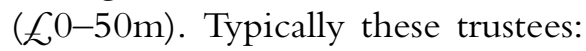

— are likely to employ only one manager

- are unlikely to have changed their fund manager in the last five years

- do not receive reports from an independent investment-monitoring service

- are looking for an improvement in the monitoring of their asset management arrangements; and

- Would in some cases prefer an expert to implement the asset management strategy.

\section{Manager change a fraught process}

For trustees dissatisfied with the performance of their fund, the decision to change a manager is fraught with difficulties. In lay terms it is a 'damned if you do, damned if you don't' dilemma. The reasons are as follows.

First, the transition costs are usually substantial, although it can be argued that with professional transition management these charges can be reduced significantly and performance maintained throughout the transition period. Transition management is a robust product in its own right in the US but as yet it represents an under-used service in the UK.

Secondly, experience demonstrates time and time again that there is a very strong likelihood that trustees will sack the manager whose performance is just about to improve, and hire the manager whose performance is poised to plummet.

As the leading performance measurer, The WM Company observed: 'Real pain will set in for those funds which appoint a manager at the top of his cycle and 
remove a manager who then turns performance around.'

\section{Trustee attitude to new asset management structures}

When the results of the survey were compared with the manager research for this report, a discrepancy was identified between what the trustees say they want and what the investment consultants say they think the trustees want.

According to the survey, trustees of small- to medium-sized schemes in particular are keen to see a broader investment consultancy service. They want more information about different management structures and the opportunity for the consultant to take a more hands-on role.

However, consultants generally argue that trustees are very conservative and are reluctant to go beyond the traditional boundaries for asset management structures.

There is a lot of truth in this. Trustees are understandably wary of pursuing a course of action that is radically different from their peers for fear of recrimination if it goes wrong.

Certainly, the consultants have had a struggle persuading trustees to move from balanced to fixed benchmarks, and from single- to multi-manager structures. However, most consultants now report that trustees are more open minded towards the concept of specialist managers and the limited use of multi-asset mandates.

For their part trustees might consider how well prepared their consultant is to advise on specialist mandates and how well the consultant actually researches these managers. If the consultant is urging caution for the genuine good of the trustees and the fund, this is perfectly reasonable. Less acceptable, however, is the prospect of consultants urging caution in order to restrict trustee choice to what services the consultant can offer, rather than what is available in the open market.

\section{The benefits of multi-manager, manager of managers}

The application of more sophisticated asset management structures may help smaller funds achieve:

- a level of risk which is fully understood and accepted by the trustees and is commensurate with the scheme's statement of investment principles (Sip)

- a reduction in short-term volatility

- a reduction in the time trustees have to spend on asset management issues; and

- swift implementation of changes in the asset management structure.

Multi-manager, multi-style funds, particularly where they are run on a MoM basis, provide the potential for robust long-term performance while simultaneously managing the risks of short-term volatility. If trustees want to satisfy the regulators and scheme members that they have taken every possible step to discharge their duty to manage investment risk, these asset management structures may prove far more satisfactory than simply choosing one or two balanced managers.

\section{Good implementation processes essential}

Not all underperformance is caused by poor investment management. Where the trustees do not have a good governance process in place the fund will suffer losses due, in particular, to poor transition management. These losses cannot be repaired with better asset management. 
The costs of changing manager must be addressed by trustees and the appointment of a formal, third-party transition manager considered. The WM Company has highlighted the substantial costs of changing manager where transition management is not handled effectively.

What is not clear from WM's research is to what extent the cost of change is due to poor implementation. In a 1997 study of the Australian scheme for Lend Lease Corporation, Coopers \& Lybrand (now PWC) found that out-performance was being lost due to ineffective implementation of specialist structures. The following year William M Mercer confirmed these views and reported that there were long delays in firing managers with organisational problems and that the fund suffered unplanned exposure to style biases.

Transition management needs to develop as a robust product in its own right in the UK as it is in the US. BGI, Mercury Asset Management, Legal \& General, State Street and Salomon already offer this product to UK pension funds, as do several consultants, including Frank Russell, and Towers Perrin.

Again, costs and efficiency need to be examined, particularly the performance of the fund during the transition process the Achilles' heel of manager changes.

\section{Can asset management be 'delegated' in deed if not in name?}

The Pensions Act 1995 insists that trustees cannot delegate their responsibility for the asset management of the fund. The important point here, however, is the level of control trustees want over the manager appointments.

Pension lawyers state that there is no specific legal definition of 'delegation'. However, there is a distinction, which the Act appears to make, between delegation and abrogation or abdication - both of which imply, in lay terms, that the trustees have washed their hands of the entire responsibility.

Historically, the trustees of UK pension funds that have adopted a multi-manager structure have retained control over which investment managers actually manage the assets.

With a 'manager of manager' service, such as that offered by Northern Trust, Frank Russell, SEI, Stamford Associates and Escher UK, among others, the trustees effectively outsource or subcontract to a third party the decision to appoint the investment managers.

Providers of MoM argue that there is no conflict of interests here and that to delegate to a third party which has demonstrable professional skills is a clear discharge of the trustee's responsibility under the Act.

Nevertheless, it is important for 'manager of manager' arrangements to be set on a firm footing under UK law and regulation. One possibility is for the trustees to waive the right to have discretion over the investment manager appointments set out in the trust deed.

Another is for the MoM provider to be registered as a discretionary manager under IMRO, as is the case with Northern Trust. Under the Pensions Act trustees are within their rights to delegate the responsibility for investment management to an IMRO-registered investment manager.

\section{Global custody service contract - a blueprint for MoM?}

A more obvious alternative is to change the Act! This is not as outrageous as it sounds. Indeed, there is a precedent for this in the service agreement between the trustees and the global custodian.

Global custodians use a network of 
sub-custodians. Under the original wording of the Pensions Act, trustees were held responsible for the sub-custodians even though they had no control over their appointment and dismissal.

This issue was resolved by an amendment to the Act that allows trustees to have a formal agreement with the chief custodian who in turn accepts responsibility for the sub-custodians.

The global custodian may act as guarantor in some but not all world markets. However, where the custodian cannot offer a guarantee it sets out its responsibility in the letter of appointment with the trustees. A similar agreement could be reached between MoM managers and trustees and set out in writing with particular reference to specific briefs — for example, satellite managers whose performance is likely to prove very volatile.

\section{Trustee liability for performance}

Good investment governance is vitally important to trustees if they wish to restrict their liability for investment performance. This is particularly true of the defined contribution (DC) market.

Until recently DC schemes and plans were not subject to the same level of scrutiny as their defined benefit (DB) counterparts. Today they face much more rigorous regulation.

Trustees are aware that unlike DB schemes, the DC structure does not pool risk between the employer and employee nor between different generations of members. As far as the scheme member is concerned what you see is what you get - however small or large the individual pot of money.

The performance, therefore, has a much more immediate impact on member satisfaction. It also raises concern about the responsibility of trustees to members who are unhappy with their fund's performance.

In the United States members of DC schemes have taken trustees to court where they felt that the trustees had failed to discharge their investment responsibilities satisfactorily. The implications for UK trustees are very worrying.

If the members complain that performance is unsatisfactory they can take the case to Occupational Pensions Regulatory Authority (Opra), the Pensions Ombudsman or, in the case of group personal pensions, to the Financial Services Authority Ombudsman.

However, all of these authorities said that ultimately such a complaint would be a question for the courts to decide.

Precedents in the United States and elsewhere indicate that the courts will support the trustees if they can demonstrate good investment governance systems are in place. These systems need to be robust and set out in formal documentation to make it clear that trustees have done their utmost to recognise and manage investment risk.

\section{Conclusion}

With the government's determination to increase scheme membership through multi-employer and affinity group schemes, most eyes are turned towards the launch of stakeholder schemes in April 2001.

Fortunately, there is no need to speculate on what these schemes may look like. Several are already available, albeit in a different guise.

As a blueprint for a stakeholder scheme it would be difficult to improve on the model designed for chemical engineers originally by the small MoM unit owned by Sedgwick Noble Lowndes which became part of William 


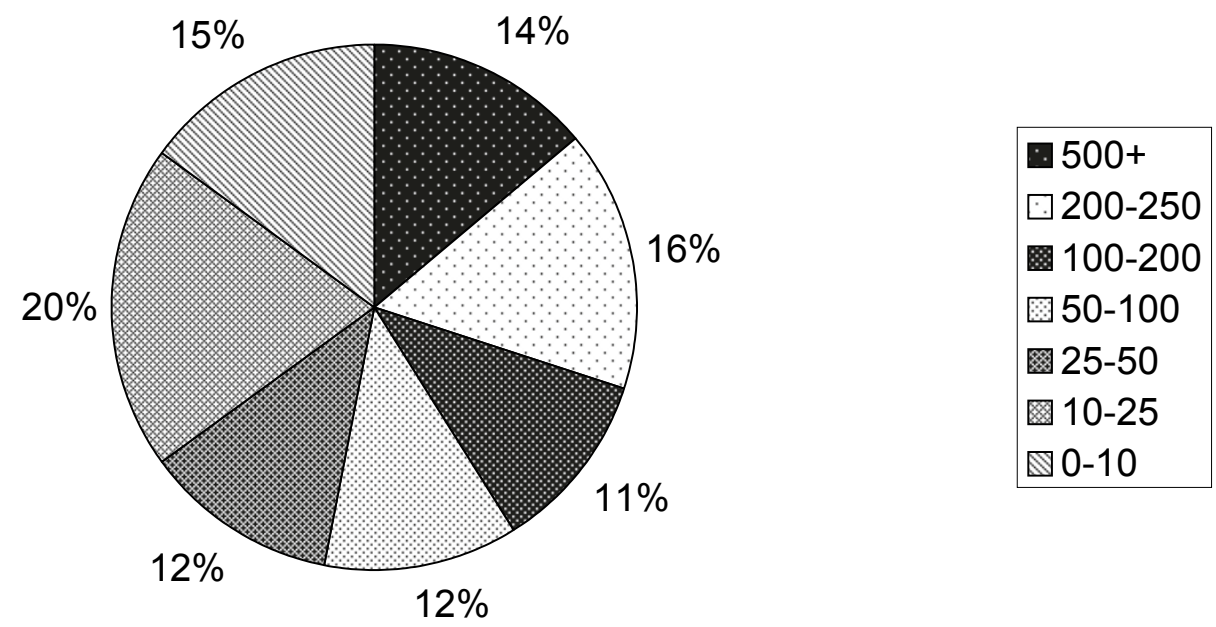

Figure 1: Overall Survey: Total size of the pension fund assets? (£m)

M Mercer when the two firms merged and was sold by Mercer to Escher UK. Escher open its doors to business this summer. The broadcasting industry also runs a potentially suitable scheme, although it is not clear whether the task of monitoring managers and implementing changes lie with the trustee, the consultant (Watson Wyatt) or the scheme provider (Prudential).

Implementation leakage could result from this uncertainty.

But the chief issue for trustees and employers planning to launch a stakeholder scheme is cost. Both the IChemE and BBC schemes were designed before the stakeholder 1 per cent annual maximum charge was proposed and, while they appear to offer good value, it is not clear whether either can reduce its costs sufficiently to become stakeholder compliant without losing external manager links.

Two schemes launched in the autumn of 1999 by the consultant Gissings and Prudential both achieved stakeholder-compliant costs, but it would appear that this is at the expense of external managers. Each scheme currently employs just one manager, and in the case of Prudential it is the provider itself.

In conclusion, it can be argued that trustees - and the Government - need to accept the fact that higher fees are justified if the multi-manager structure reduces short-term volatility - a critical issue for DC schemes.

As for the multi-style MoM model, Frank Russell suggests it costs an extra 20 basis points to provide two or three managers instead of just one in an equity fund. If this is the case then the model appears to represent good value in the light of the reduced volatility it confers.

\section{Trustee survey results}

\section{Breakdown of survey participants by size and industry sector}

The PMI questionnaires were used as part of a separate control group for the pension trustee attitude survey. Many of the PMI trustee group members are qualified and have considerable experience. The aim, therefore, was to determine whether this group would demonstrate a different attitude to key 


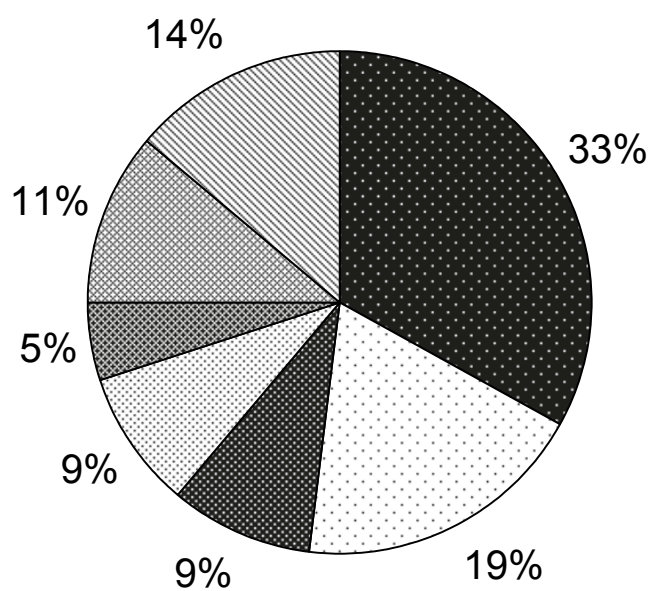

$500+$

๑200-250

100-200

图 50-100

图 25-50

図 10-25

$\mathbb{\$} 0-10$

Figure 2: PMI Participants: Total size breakdown (£m) of fund size

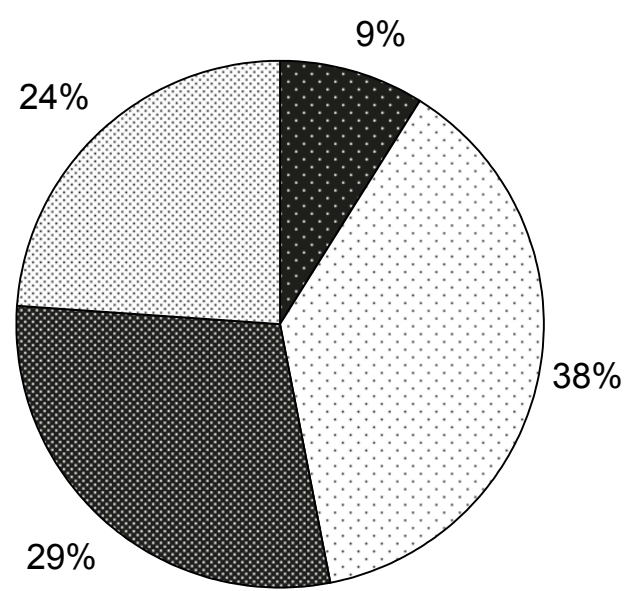

Other $\square$ Small-Cap

FTSE 250

ORTSE 100

Figure 3: Overall Survey: Sponsoring employer by index classification

factors such as risk, responsibility and level of knowledge compared with the entire range of respondents.

The results broadly matched those of the main survey except in the following cases set out below:

1. The overall size profile was different from the main survey response

Figure 1 shows that the main survey respondents represented an even spread of fund sizes, therefore providing a good representative sample. The PMI profile shown in Figure 2 had more responses in the $\mathcal{L}^{5} 500 \mathrm{~m}+$ sector.

\section{The major indices}

The overall survey showed participants represented an equal spread of indices and other/unlisted companies as shown in Figure 3.

The PMI group shown in Figure 4 had a greater proportion of trustees of funds for companies in the FTSE 100 


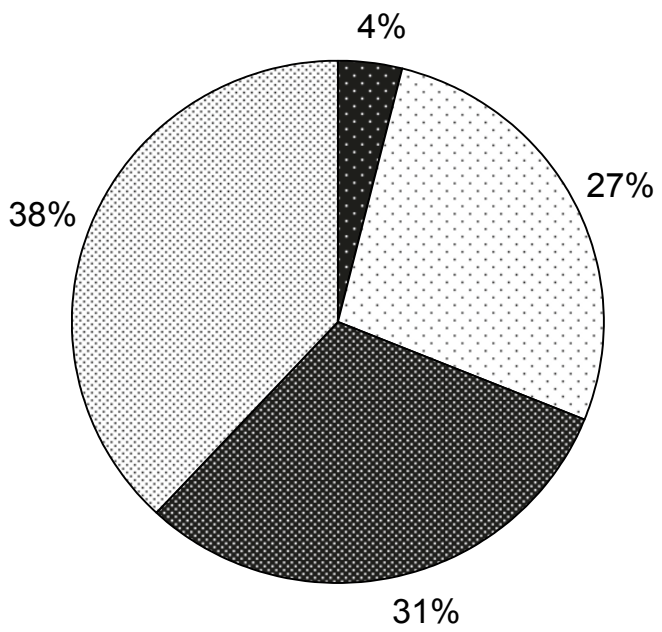

Other

Figure 4: PMI Participants: Sponsoring employers by index classification

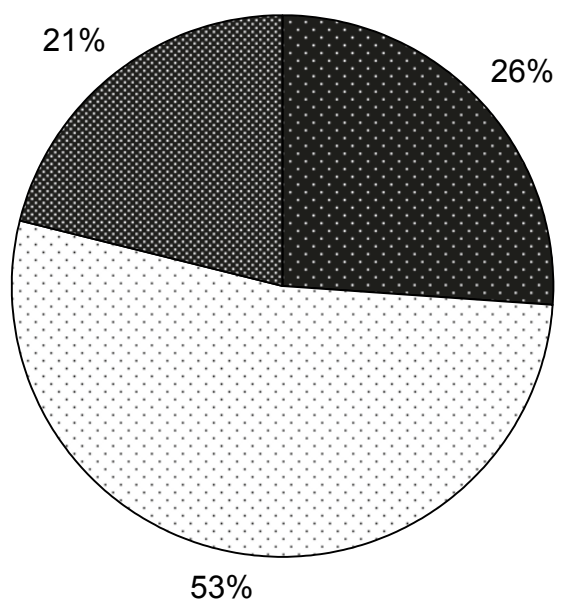

Neutra $\square$ Yes No

Figure 5: Overall Survey: Are you (as a trustee) happy with your pension scheme investment performance?

and FTSE 250 indices than the overall survey.

\section{Level of satisfaction with pension fund performance}

It was noticed that a greater number of trustees from the PMI survey said they were happy with the performance of their fund(s).

Figure 5 shows the overall survey response to performance. Figure 6 shows the PMI survey response to the same question.

4. Length of time since a fund manager had been changed

The main survey (see Figure 7) showed that the majority of funds had either changed an asset manager within the last 12 months, or had not changed the manager for over five years. 


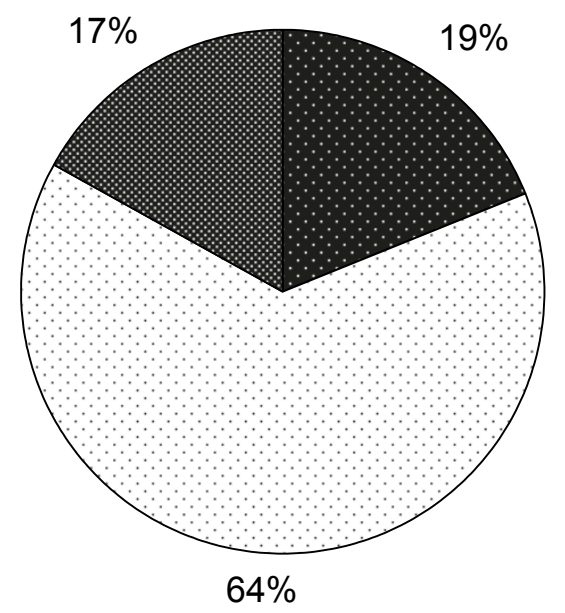

- Neutral

๑Yes

图 No

Figure 6: PMI Participants: Are you (as a trustee) happy with your pension scheme investment performance?

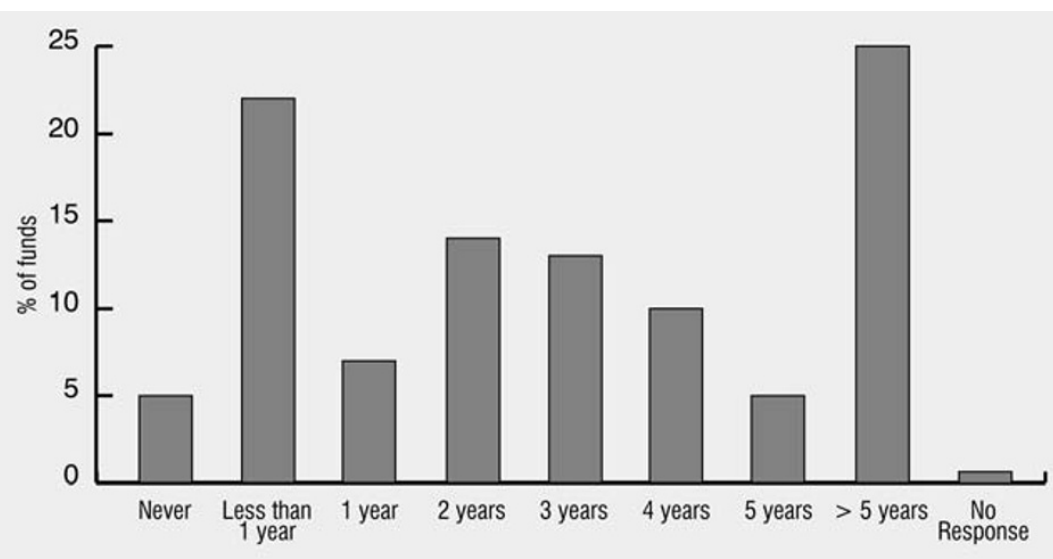

Figure 7: Question 13 - Length of time since you last changed an investment manager

In the PMI group (see Figure 8) more fund managers had been changed within the past two years than during any other period of time.

\section{Attitudes to active and passive} management

The overall survey shows 5 per cent of trustees strongly favoured passive management (see Figure 9).

The PMI response showed a greater incline towards active management with no trustees strongly favouring passive management, as shown in Figure 10.

\section{Appendix: Industry standard terminology for new manager structures}

\section{Multi-manager}

Multi-manager can be anything from two balanced managers — a strategy 


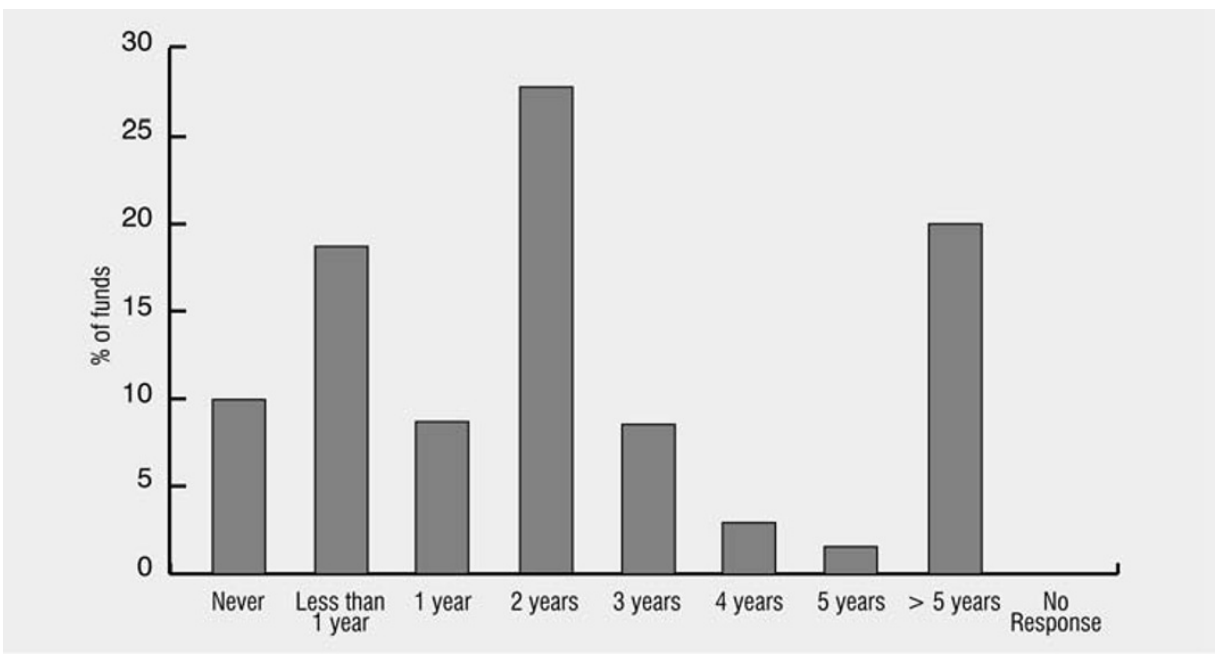

Figure 8: PMI Question 13 - Length of time since you last changed an investment manager

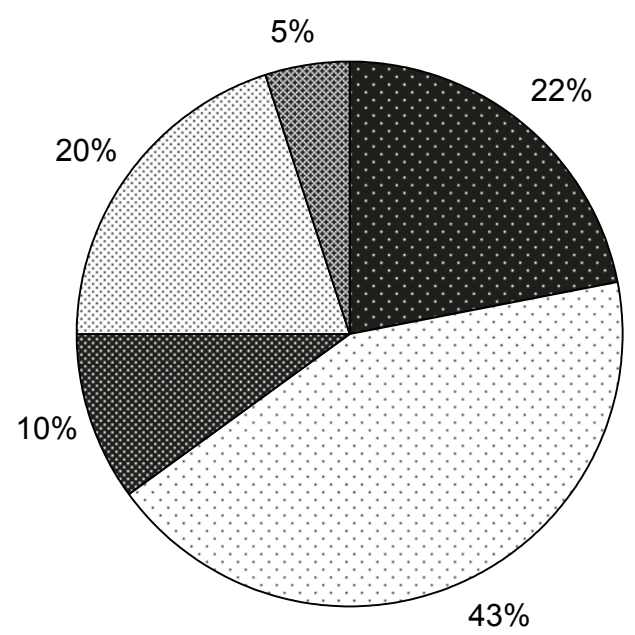

Strongly favour active management

$\square$ Inclined towards active management

No strong views or knowledge

Onclined towards passive management

Strongly favour passive management

Figure 9: Overall Survey: What best describes your atribude to active and passive investment management strategies?

adopted by many medium-sized UK pension funds - to a core index fund with satellite active specialists (core/satellite) - an approach favoured by Watson Wyatt and William $M$

Mercer. The point to remember about multi-manager is that, although the consultant advises on the appointments, the trustees retain control over who is appointed and have a direct agreement with the asset managers.

In defining different types of manager it is important to keep in mind their objective. Watson Wyatt suggests the following benchmarks for outperformance, assuming index is zero:

- Balanced 1 per cent p.a.

- Active specialist 1.5-2 per cent p.a.

- Satellite 3 per cent p.a. 


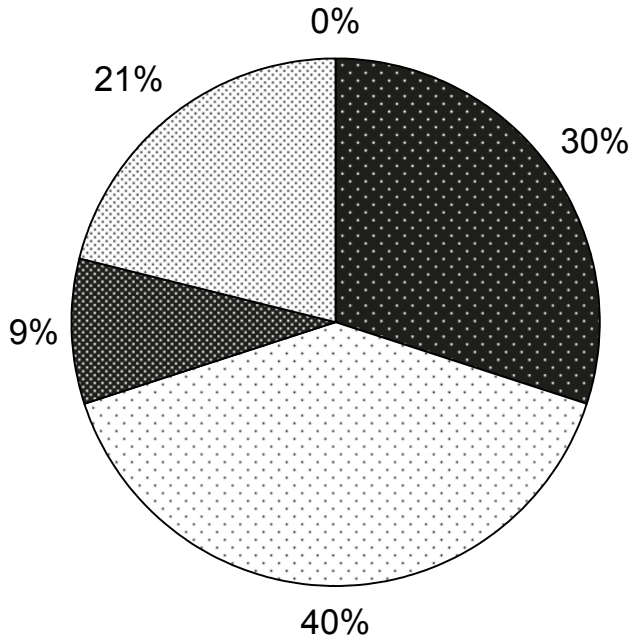

Strongly favour active
management
$\square$ Inclined towards active
management
No strong views or
knowledge
Inclined towards passive
management
图 Strongly favour passive
management

Figure 10: PMI Question 5 - What best describes your attitude to active and passive investment management strategies?

\section{Multi-style}

Multi-style is used to reduce the risk of the short-term volatility that results from style bias. This is achieved by appointing two or more managers for each asset class. The managers have different (complementary) styles so that the fund performance is maintained throughout the market cycle, whether the flavour of the season is value or growth, small or large cap, pharmaceuticals or IT. Multi-style, therefore, reduces the opportunity for exceptional short-term outperformance, but over the long term minimises risk at total fund level.

\section{Manager of managers}

As currently offered, MoM is usually directed at the institutional and high net worth individual markets and offers a range of sector funds - for example, domestic equities, overseas equities, bonds and cash. The trustees hand over to the MoM provider the responsibility of selecting, hiring, monitoring and firing the sector fund managers. The trustees usually retain control of the strategic asset allocation or direct the MoM to act in accordance with the scheme's statement of investment principles. The important point to remember is that the asset management agreement is between the trustees and the MoM provider not between the trustees and the sub-managers.

\section{Source: Informa Publishing Group,}

Tel. 01206772113

email:enquiries@informa.com

This article was first published in the Pensions Management Institute Technical News, December, 1999. 